\title{
L'oïdium américain du haricot (Erysiphe polygoni D.C.) dans la zone antillaise : recherche de géniteurs de résistance
}

\author{
C.M. Messiaen ${ }^{1}$, P. Pauvert ${ }^{2}$, G. Jacqua ${ }^{2}$ et A. Laraque ${ }^{3}$ \\ 1 INRA, Biologie et Pathologie végétale, 9, place Viala, 34060 Montpellier Cedex; \\ 2 INRA, Antilles-Guyane, Pathologie végétale et Malherbologie, BP 1232, 97184 Pointe-à-Pitre Cedex, France; \\ 3 Projet Madian-Salagnac-Aquin, BP 131, Port-au-Prince, Haîti
}

(reçu 1-8-1988, accepté le 12-12-1988)

\begin{abstract}
Résumé - L'oïdium américain du haricot semble particulièrement virulent dans la zone antillaise, où il attaque des variétés considérées comme résistantes aux Etats-Unis. Des géniteurs de résistance ont été trouvés en Haïti, parmi lesquels les lignées "Kenscoff I", "Salagnac 86", "Salagnac 131" ont été plus particulièrement utilisées dans les programmes de sélection.

L'étude de la descendance de croisements a permis de définir une "composante précoce" (résistance des plantules) et une "composante tardive" (tolérance du feuillage adulte) dans la résistance issue des géniteurs haîtiens. La première est liée à un gène dominant, la deuxième, d'hérédité polygénique, est à tendance récessive.
\end{abstract}

sélection variétale - Phaseolus vulgaris - facteurs climatiques - hérédité - Erysiphe polygoni

Summary - American bean powdery mildew in the West Indian area: identification of resistance sources. The american bean powdery mildew seems to be very virulent in the West Indian area. Resistant lines have been found in Haïti, such as "Kenscoff I", "Salagnac 86", "Salagnac 131". "Borlotto nano Vigevano" was used as susceptible check.

The study of the progeny from crosses between these cultivars allowed to distinguish two components of the resistance obtained from Haitian lines: "seedling resistance", induced by a dominant gene, and "adult plant resistance", which seems polygenic recessive.

selection - Phaseolus vulgaris - climatic factors - heredity - Erysiphe polygoni

\section{Introduction}

Notre intérêt pour l'oïdium américain du haricot, Erysiphe polygoni D.C. (différent de l'oïdium que l'on observe parfois en Europe sur haricot sous serre provoqué par Erysiphe cichoracearum D.C.), a débuté dans les années 70 . Cette maladie se révélait importante sur cette plante en Guadeloupe, ainsi que le plus grave obstacle à la production en Haiti de haricots à grains rouges panachés (les plus estimés).

\section{Historique}

On trouve peu de références récentes sur cet oïdium. Schwartz et al. (1981), en Colombie, tirant parti d'une forte attaque sur un essai variétal comportant des parcelles traitées et non trai- tées, font état de baisses de rendement allant de 2 à $69 \%$ suivant les cultivars et signalent comme résistants «Porrillo sintético» (grain noir) et quelques numéros "BAT». Maxfield (1968) signale une aggravation de la sensibilité de «Bountiful», de l'hypersensibilité de «Pinto» à l'oïdium quand les plantes sont attaquées par le Southern bean mosaic virus. Dans les années $50-60$, on trouve cet oïdium signalé dans un certain nombre de pays, ou utilisé comme champignon-test pour des essais de fongicides en serre (Miller, 1952).

II faut remonter jusqu'aux publications de Dundas $(1936,1941,1942)$ pour trouver une étude approfondie sur la résistance à l'oïdium américain du haricot. Se basant soit sur des observations en champ, soit sur des essais réalisés avec 
des feuilles en boîte de Pétri, cet auteur définissait les températures cardinales du parasite (développement très lent à $8^{\circ} \mathrm{C}$, optimal à $20^{\circ} \mathrm{C}$, croissance réduite à $25^{\circ} \mathrm{C}$ et nulle à $30^{\circ} \mathrm{C}$ ). II mettait par ailleurs en évidence un gène dorninant de résistance chez un certain nombre de variétés, dont «Pinto". Des variétés d'un niveau de résistance intermédiaire lui permettaient, en 1941 , de décrire 3 races et, semble-t-il, 14 en 1942, «Pinto» résistant à 12 d'entre elles. Malheureusement l'«abstract" de 1942 n'a jamais été développé en un article complet.

Par la suite, un certain nombre de stations de sélection de Floride et de Californie ont signalé l'obtention de variétés de haricot résistantes, comme les mange-tout "Contender» «Florida belle», «Florida white wax», «Full green», «Sérninole». En 1945, Magoon affirmait que “'oïdium n'est plus un danger, puisque toutes les nouvelles variétés développées par I'USDA lui sont résistantes". Aujourd'hui encore, certains catalogues américains font état de la résistance de "Contender", "Extender", "Provider" à de «nombreuses races d'oïdium».

\section{Travaux réalisés en Guadeloupe dans les années 70}

Pauvert et Jacqua (1972) observaient l'oïdium sur un certain nombre de légumineuses sauvages (Pueraria phaseoloides, Centrosema pubescens, Vigna lutoela, Cassia tora), cultivées traditionnellement (Phaseolus vulgaris, Vigna unguiculata), ou récemment introduites ( $V$. unguiculata var. sesquipedalis, $V$. mungo, $V$. radiata).

Dans une étude de gammes d'hôtes en serre, ils désignaient :

- comme sensibles : les $P$. vulgaris "Contender", «St Fiacre», les $V$. unguiculata «Black eye» et "sesquipedalis ", les $V$. mungo et radiata;

- comme peu sensibles : le Phaseolus lunatus «Florida butter speckled», le Pisum sativum «Mange-tout hâtif à large cosse», le Vigna angularis «Adzuki»;

- comme résistants : les "pois boucoussou" (Lablab niger ), le Pisum sativum «Manoa sugar».

Contrairement à celui des cucurbitacées, l'oïdium du haricot ne semble pas manifester de phénomènes de résistance au bénomyl. C'est peut-être l'usage généralisé de ce fongicide qui a fait disparaître le problème "oïdium du haricot" aux Etats-Unis. La recherche de géniteurs de résistance à l'oïdium fait l'objet du travail présenté dans cette publication.

\section{Matériel et Méthodes}

\section{Matériel végétal}

Les géniteurs de résistance ont été cherchés en Haïti. C'est en 1972 que, parmi 15 lignées extraites d'une poignée de haricots achetés sur le marché de Kenscoff, la lignée "Kenscoff I" se révéla résistante à l'oïdium en Guadeloupe. En décembre 1976, Bannerot (INRA, Versailles), au cours d'une mission en Haïti, conseilla à V. de Reynal de marquer dans une parcelle paysanne d'environ $3000 \mathrm{~m}^{2}$ (150 000 plantes), quelques individus dont le feuillage paraissait exceptionnellement sain. Trois cent cinquante lignées furent ainsi isolées et envoyées en Guadeloupe, où elles furent soumises à inoculation en serre. Sept lignées furent repérées comme résistantes dès le stade "2 feuilles» et conservées pour évaluation de la sensibilité du feuillage adulte. "Contender» (témoin sensible) étant noté “ 10 ", nous obtenions à 45 jours, les notes suivantes (Tableau I).

Tableau I. Evaluation de la sensibilité du feuillage adulte.

Lignées Notes

$\begin{array}{lll}\text { Salagnac } 131 & 0 \\ \text { Salagnac } 86 & 1 \\ \text { Salagnac } 120 & 1 \\ \text { Salagnac } 126 & 2 \\ \text { Salagnac } 81 & 5 \\ \text { Salagnac } 89 & 5 \\ \text { Salagnac } 92 & 6\end{array}$

Nous aurions pu, déjà à cette époque, déceler une "composante précoce» et une "composante tardive» de la résistance. On peut regretter l'élimination des 343 lignées "sensibles au stade 2 feuilles», dont certaines auraient pu se montrer hautement tolérantes sur feuillage adulte. Le très haut niveau de résistance de "Salagnac 131", le très bon type variétal de "Salagnac 86" (plantes naines, gros grains panachés rouges), nous ont fait choisir ces lignées comme géniteurs dans nos programmes de sélection, sans oublier "Kenscoff l" ni sa version à grains rouges panachés "Kenscoff super», obtenue par rétrocroisement.

Nous avons aussi utilisé une autre lignée haïtienne "Nicanor 2.4." (sensible au stade 2 feuilles, grain noir), et parmi les lignées issues de nos programmes de sélection «Salagnac 59" (grain noir), «Salagnac 90" (grain rouge panaché) et "CNR44" (grain rouge uni), dont la généalogie est la suivante : Salagnac 59 est issu de : B789 x Salagnac 131 (B 789 est une lignée haïtienne au grain noir, isolée par Bannerot sur le marché de Petite-Rivière-de-Nippes). Salagnac 90 a été sélectionné pour une plus vive coloration du grain par rapport à Salagnac 86, à partir des croisements [(Constanza $1 \times$ Kenscoff I) $\times$ Salagnac 86] $\times$ Salagnac 86) “Constanza 1" étant une variété provenant de la République dominicaine). CNR44 est issu de [(Salagnac $86 \times$ Epicerie de Sault) $\times$ Kenscoff super] $\times$ (Sca- 
biola rossa x Red Kidney), “Epicerie de Sault» étant une lignée volubile à gousse rouge vif uni isolée dans le Midi de la France, sensible à l'oïdium, au même titre que "Scabiola rossa" ou "Red Kidney".

\section{Sources d'inoculum}

La variété italienne «Borlotto nano vigevano» (plantes naines, grain blanc rayé de rouge) a été choisie comme support de l'oïdium, à cause de sa très grande sensibilité. Des feuilles naturellement infectées de haricot ou de Pueraria phaseoloides sont suspendues au dessus de plantules de “Borlotto" et maintenues une vingtaine de jours au stade 2 feuilles par excision du bourgeon terminal, à raison de 4 plantules par pot de $10 \mathrm{~cm}$. Tous les 15 jours, de nouvelles plantules sont disposées à côté des anciennes, de telle façon que le vent d'est dissémine les conidies dans leur direction (les serres sont largement ouvertes et, en Guadeloupe, le vent souffle presque toujours de l'est). On peut aider le vent en soufflant chaque matin sur la "source d'inoculum".

Pour des observations sur de plus longues durées (jusqu'à la floraison ou au-delà), nous laissons les plantes se développer normalement, avec 4 plantes par pot de $20 \mathrm{~cm}$.

Les essais au champ sont effectués en semis de lignes espacées de $90 \mathrm{~cm}$, les grains étant espacés de $5 \mathrm{~cm}$ sur la ligne. Les contaminations sont réalisées en intercalant des lignes de "Borlotto» ou en mettant cette même variété à l'est du champ, ce qui crée un gradient de maladie, compte tenu de la régularité du vent.

\section{Résultats}

Comportement de l'oïdium en serre suivant les saisons

Après un démarrage parfois irrégulier en octobre, on obtient, à partir du 15 novembre, un développement très rapide de l'oïdium sur «Borlotto" et sur les variétés de sensibilité comparable. Dès le $7 \mathrm{e}$ jour après la germination, on observe sur les 2 premières feuilles des taches vert sombre formant une mosaïque qui, dès le $12^{\mathrm{e}}$ jour, deviennent des colonies poudreuses confluentes. On observe un développement analogue de l'oïdium sur les feuilles suivantes, qui peuvent prendre un aspect cloqué, du fait de l'invasion par le parasite avant que la feuille n'ait atteint sa taille définitive.

Cette "manifestation rapide" de l'oïdium se poursuit en général jusqu'à début avril, ce qui correspond à la période où les maxima sous serre ne dépassent pas $30^{\circ} \mathrm{C}$. Ensuite, les maxima supérieurs à $30^{\circ} \mathrm{C}$ deviennent plus fréquents et le développement de l'oïdium plus lent. Les colonies n'apparaissent pas avant que la feuille ait atteint sa taille définitive et mettent plus de temps à confluer. La production de conidies est moins abondante. Cette période de «manifesta- tion lente» de l'oïdium dure jusqu'à octobrenovembre.

\section{Comportement de l'oïdium au champ}

Par rapport à ce qui se passe en serre, non seulement les journées chaudes, mais aussi les périodes excessivement pluvieuses (mois à plus de $300 \mathrm{~mm}$ ) contrarient le développement de l'oïdium. En Guadeloupe, les essais les plus réussis seront donc ceux dont la période de végétation comprendra l'intervalle 10 février-20 mars, période assez régulièrement la plus fraîche et la plus sèche. On peut voir réapparaître l'oïdium, après les pluies de mai, si des périodes sèches se produisent en juin-juillet. II disparaît à peu près complètement des parcelles cultivées en septembreoctobre-novembre (fortes pluies). II se conserve alors sur des légumineuses sauvages, par exemple Pueraria phaseoloides à l'abri de grands arbres.

En Haïti, à 700-900 m d'altitude, parmi les trois semis traditionnels, celui d'octobre est en général très attaqué, celui de février-mars un peu moins, celui de juillet, soumis à de très fortes pluies, voit l'oïdium passer au second rang par rapport à la rouille.

\section{Hérédité de la résistance à l'oïdium}

\section{Comportement en serre et au champ de quelques variétés}

Dans le tableau II, nous mentionnons pour quelques variétés :

- leur comportement précoce en serre (12e jour) en période de «manifestation rapide» de l'oïdium. On peut délimiter 2 classes très distinctes, plantules sensibles, plantules résistantes, notées "S" et «R»;

- leur comportement en serre au 45e jour (début de grossissement des gousses), noté selon une échelle de 0 (résistance totale) à 10 (sensibilité de type «Borlotto");

- pour certaines d'entre elles, leur comportement au champ en semis de janvier 1987, noté avec une échelle identique.

Nous retrouvons «Salagnac 86 » et «Salagnac 81 " classés dans le même ordre qu'en 1976. On n'observe aucune liaison entre le comportement précoce "résistant" ou "sensible" des variétés et leur comportement tardif, en serre ou au champ. La notation tardive en serre pénalise dans une certaine mesure "Kenscoff super" et «Nicanor 2.4.».

\section{Hérédité des composantes de la résistance}

Nous avons observé le comportement de 43 plantes F2 issues du croisement : Salagnac $86 \times$ 
Tableau II. Comportement de quelques variétés en serre et au champ vis-à-vis de l'oïdium (Notes de 0 à 10). R : plantules résistantes; $\mathrm{S}$ : plantules sensibles.

\begin{tabular}{|c|c|c|c|c|c|}
\hline & & Serre $12 j$ & Serre $45 j$ & Champ $20 j$ & Champ 50 \\
\hline Salagnac & 131 & $\mathrm{R}$ & 0,6 & - & - \\
\hline Salagnac & 59 & $\mathrm{R}$ & 1,2 & 0,5 & 0 \\
\hline Salagnac & 86 & $\mathrm{R}$ & 2,5 & - & - \\
\hline Kenscoff sup & per & $R$ & 5 & 0,5 & 0 \\
\hline Salagnac & 81 & $\mathrm{R}$ & 7,5 & - & - \\
\hline CNR44 & & $\mathrm{R}$ & 8,5 & 2,5 & 7 \\
\hline Nicanor & 2.4 . & $\mathrm{S}$ & 2,5 & 2 & 0 \\
\hline Salagnac & 90 & $s$ & 2,5 & 2 & 1 \\
\hline Borlotto & $n v$ & $S$ & 10 & 4 & 9 \\
\hline
\end{tabular}

Borlotto nano vigevano, en période de «manifestation rapide" de l'oïdium (notations aux $12^{\mathrm{e}}$ et $45^{\mathrm{e}}$ jours). A la première notation, nous avons trouvé 8 plantules sensibles et 35 résistantes. A la seconde notation, en répartissant les plantes en 4 classes $(0$ à 2,5; 2,5 à 5,$5 ; 5,5$ à 7,$5 ; 7,5$ à 10), nous avons observé la distribution suivante :

- classe $1: 5$ plantes (dont une notée «S» précocément);

- classe $2: 6$ plantes;

- classe $3: 8$ plantes (dont 3 notées «S" précocément);

- classe $4: 24$ plantes (dont 4 notées «S» précocément).

On ne retrouve donc en notation tardive que $11,6 \%$ de plantes d'un niveau de résistance analogue à celui de "Salagnac 86 ». II n'existe pas, là non plus, de relation entre les comportements précoce et tardif des plantes. Pour la "résistance» précoce, la proportion observée (8/43) n'est pas en contradiction avec l'hypothèse d'un gène dominant. Nous avons obtenu des disjonctions satisfaisantes dans les F2 des croisements : CNR1 x Avermelhado (lignée sœur de CNR44, et variété brésilienne sensible au stade plantule, assez tolérante au stade adulte) et $(223 . \mathrm{V} \times 63) \times$ Salagnac 59 (croisement réalisé en vue d'obtenir des mange-tout volubiles résistants à l'oïdium. «223.V» et «63» sont sensibles).

En revanche, la répartition en classes pour la résistance tardive, aussi bien en serre pour Salagnac $86 \times$ Borlotto en F2 que pour les familles F3 au champ des 2 autres croisements, suggère une hérédité polygénique à tendance récessive. Dans des descendances de croisement entre géniteurs considérés comme sensibles, nous avons observé parfois au champ des familles F3 ou F4 "transgressives" pour la tolérance (familles notées «5» issues de parents notés «8» ou «9»). II est probable que certains des gènes participant à la «résistance tardive» sont discrètement présents à l'état isolé dans des variétés "sensibles".

Un test peu encombrant pour évaluer en serre le niveau de résistance de variétés ou de descendances de croisement

Nous avons mentionné plus haut la possibilité de miniaturiser des plants de haricot en procédant à l'ablation précoce des 2 premières feuilles. Nous avons pratiqué cette opération sur les variétés suivantes, en période de "manifestation rapide» de l'oïdium :

Salagnac 131

Salagnac 86

CNR 44

Salagnac 90 pourvues du gène dominant

Nicanor 2.4

Borlotto n.v.

Quatorze jours après le semis, on peut observer sur la première feuille trifoliée le symptôme caractéristique "taches vert foncé en mosaïque", sur les variétés dépourvues du gène de résistance. Trente jours après le semis, on peut observer sur cette même feuille un oïdium abondant et poudreux sur «Borlotto". En revanche, il est moins abondant sur "Nicanor 2.4." et a presque complètement régressé sur «Salagnac 90 ». Au contraire, "CNR44" présente un assez abondant développement de l'oïdium sur la première feuille trifoliée. La deuxième feuille trifoliée est pratiquement indemne d'oïdium sur "Nic 2.4." et "Salagnac 90", sérieusement atteinte chez "CNR44", abondamment infectée chez "Borlotto". "Salagnac 86 » et «Salagnac 131 » restent pratiquement indemnes pendant toute la durée de l'expérience. On peut donc ainsi obtenir, en 30 jours, sur plantes miniaturisées, un résumé complet de leur comportement vis-à-vis de l'oïdium. II est 
possible, après le $30 \mathrm{e}$ jour, de repiquer (avec la motte) les plantes résistantes dans de plus grands pots et d'en obtenir une ou deux gousses.

\section{Conclusion}

Les souches antillaises de l'oïdium américain du haricot sont sans doute plus virulentes que celles de Floride ou de Californie, comme le montrent les sensibilités totales de "Contender» en Guadeloupe ou de "Pinto" en Haïti. En revanche, la constance des réactions au champ de 1976 à 1987 des lignées caractéristiques de nos collections, en Guadeloupe et Haîti, la stabilité du comportement des géniteurs de résistance haïtiens peuvent donner confiance dans l'usage de ces derniers dans les programmes de sélection.

Par ailleurs, les phénomènes de "manifestation rapide» ou "manifestation lente» de l'oïdium en fonction des saisons, de "résistance précoce» ou "résistance tardive» chez les plantes, ont été observés avec un inoculum non cloné. Dans les serres largement ouvertes sur l'extérieur cohabitaient, avec les plantes-hôtes constituées par des plantules de "Borlotto", des plantes plus âgées $F_{1}$ et $F_{2}$ de niveaux de résistance très variés pouvant elles aussi constituer une source d'inoculum. Nos expériences ne permettent donc pas de discerner si les phénomènes observés correspondent à une modulation du comportement d'un couple hôte-parasite homogène par la température et l'âge de l'hôte, ou à une hétérogénéité de l'inoculum.

\section{Remerciements}

L'étude sur l'oïdium du haricot a pu être approfondie à I'INRA - Antilles-Guyane en 1985, 1986 et 1987, grâce à l'aide de la Commission des Communautés européennes (contrat "Légumineuses alimentaires", TSDA073F.).

Nous avons évoqué le rôle joué par $\mathrm{H}$. Bannerot et V. de Reynal dans la découverte de lignées résistantes à l'oïdium en Haïti. Par la suite, P. Lucas (coopérant français) et $P$. Mathieu (agronome haïtien) ont participé aux travaux de sélection du haricot en Haïti, avant la reprise du sujet par A. Laraque.

\section{Références}

Dundas B. (1936) Inheritance of resistance to powdery mildew in beans. Hilgardia 10, 241-253

Dundas B. (1941) Further studies on the inheritance of resistance to powdery mildew in beans. Hilgardia 13, $551-565$

Dundas B. (1942) Breeding beans for resistance to powdery mildew and rust. Phytopathology 32, 828 (Abstract)

Magoon C.A. (1945) Breeding better vegetables for the south at the US regional breeding lab. Misc. Publ. USDA 578

Maxfield J.E. (1968) Investigations under controlled environment conditions on interaction between Erysiphe polygoni D.C. and Southern bean mosaic on beans var. Bountiful and Pinto. Diss. abstr. 28, 7, Bi286

Miller H.J. (1952) A method for obtaining high incidence of powdery mildew on snap beans in the greenhouse for fungicide screening tests. (Abstract) Phytopathology 42, 114

Pauvert P. \& Jacqua G. (1972) Lutte contre l'oïdium des légumineuses par traitement des semences au bénomyl. Nouvelles maraîchères et vivrières. INRACRAAG, 3

Schwartz H.F., Katherman M.J. \& Thung M.D. (1981) Yield response and resistance of beans to powdery mildew in Colombia. Plant Dis. 65, 737-738 